\title{
85683 - OXIDO NÍTRICO COMO BIOMARCADOR ASSOCIADO A HIPERTENSÃO ARTERIAL EM IDOSOS RIBEIRINHOS DA AMAZÔNIA
}

\author{
Pôster - Gerontologia
}

\author{
Nathália Cardoso de Afonso Bonotto / Bonotto, NCA / UFSM; Fernanda Barbisan / Barbisan, F / UFSM; \\ Ednea Aguiar Maia-Ribeiro / Maia-Ribeiro, EA / UFSM; Euler Esteves Ribeiro / Ribeiro, EE / UFSM; \\ Bárbara Osmari Turra / Turra, BO / UFSM; Ivana Beatrice Mânica da Cruz / Cruz, IBM / UFSM
}

Introdução: Células endoteliais sintetizam o óxido nítrico $(\mathrm{ON})$, responsável pelo relaxamento e contração da parede arterial. A disfunção endotelial contribui para um desbalanço na produção de $\mathrm{ON}$, e pode contribuir com o desenvolvimento de vários fatores de risco de doenças cardiovasculares como a hipertensão arterial sistêmica (HAS), que está relaciona com a idade, por isso apresenta maior prevalência em idosos, bem como o aumento no estresse oxidativo. Objetivos: Analisar a relação entre ON, um biomarcador plasmático do estresse oxidativo, com os níveis elevados de pressão arterial sistêmica em idosos ribeirinhos da Amazônia. Metodologia: Selecionou-se 593 idosos (275 homens, 318 mulheres), inseridos na Estratégia de Saúde da Família (ESF-SUS) do município de Maués-AM com idade média de 72,28 \pm 8,05 anos. Foi aplicada entrevista estruturada para avaliar a história clínica e estilo de vida. $\mathrm{O}$ aferimento da pressão arterial sistólico-diastólica (PAS/PAD), foi realizada por dois profissionais da saúde em dois momentos distintos, seguindo as instruções da V Diretriz Brasileira de Hipertensão Arterial. Os níveis de ONp foram medidos através da quantificação plasmática espectrofotométrica dos níveis de nitrato e nitrito. O estudo foi aprovado pelo Comitê de ética em Pesquisa da Universidade Federal de Santa Maria, Resultados: Os níveis médios de ON foram de 32,82 $\pm 26,46 \mathrm{mmol} / \mathrm{mL}$. Foi usado o valor do percentil 75 para comparação entre hipertensão grupo homens $(41 \mathrm{mmol} / \mathrm{mL})$ e mulheres $(45 \mathrm{mmol} /$ $\mathrm{mL}$ ). Nos homens os níveis elevados de PAS (> $140 \mathrm{~mm} / \mathrm{Hg}$ ) foram associados a níveis elevados de ON, independente da idade, história de hipertensão, diabetes, obesidade e outras doenças cardiovasculares. Nas mulheres esta associação não foi observada. Conclusão: Os resultados sugerem que níveis elevados de ON podem indicar em homens idosos com HAS não controlada, independente de outros fatores de risco cardiovascular. 